\title{
A flexible scintillation light apparatus for rare event searches
}

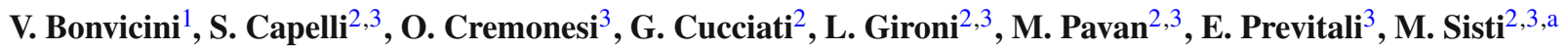 \\ ${ }^{1}$ INFN-Sezione di Trieste, 34149 Trieste, Italy \\ 2 Dipartimento di Fisica, Università di Milano-Bicocca, 20126 Milan, Italy \\ ${ }^{3}$ INFN-Sezione di Milano-Bicocca, 20126 Milan, Italy
}

Received: 24 July 2014 / Accepted: 22 October 2014 / Published online: 6 November 2014

(C) The Author(s) 2014. This article is published with open access at Springerlink.com

\begin{abstract}
Compelling experimental evidences of neutrino oscillations and their implication that neutrinos are massive particles have given neutrinoless double beta decay $(\beta \beta 0 v)$ a central role in astroparticle physics. In fact, the discovery of this elusive decay would be a major breakthrough, unveiling that neutrino and antineutrino are the same particle and that the lepton number is not conserved. It would also impact our efforts to establish the absolute neutrino mass scale and, ultimately, understand elementary particle interaction unification. All current experimental programs to search for $\beta \beta 0 v$ are facing with the technical and financial challenge of increasing the experimental mass while maintaining incredibly low levels of spurious background. The new concept described in this paper could be the answer which combines all the features of an ideal experiment: energy resolution, low cost mass scalability, isotope choice flexibility and many powerful handles to make the background negligible. The proposed technology is based on the use of arrays of silicon detectors cooled to $120 \mathrm{~K}$ to optimize the collection of the scintillation light emitted by ultrapure crystals. It is shown that with a $54 \mathrm{~kg}$ array of natural $\mathrm{CaMoO}_{4}$ scintillation detectors of this type it is possible to yield a competitive sensitivity on the half-life of the $\beta \beta 0 v$ of ${ }^{100} \mathrm{Mo}$ as high as $\sim 10^{24}$ years in only 1 year of data taking. The same array made of ${ }^{40} \mathrm{Ca}^{\text {nat }} \mathrm{MoO}_{4}$ scintillation detectors (to get rid of the continuous background coming from the two neutrino double beta decay of ${ }^{48} \mathrm{Ca}$ ) will instead be capable of achieving the remarkable sensitivity of $\sim 10^{25}$ years on the half-life of ${ }^{100} \mathrm{Mo} \beta \beta 0 v$ in only 1 year of measurement.
\end{abstract}

\section{Introduction}

In this paper we propose a novel approach to neutrinoless double beta decay searches which uses high performance

\footnotetext{
a e-mail: monica.sisti@mib.infn.it
}

solid state detectors to read the scintillation light emitted by large mass crystals with high energy resolution.

Neutrinoless double beta decay is an extremely rare phenomenon hypothesized long time ago but never observed. Its discovery would be a major breakthrough in astroparticle physics, since it would demonstrate the lepton number non-conservation and unveil the Majorana character of the neutrino, i.e. prove that the neutrino is equal to its own antiparticle. At the same time it would allow to assess the absolute neutrino mass scale with high sensitivity and would help point us towards the proper extension of the Standard Model of Particle Physics. This justifies the large number of experimental programs devoted to the search of this decay with different techniques. On the other hand, all these programs are facing with the technical and financial challenge of increasing the experimental mass while maintaining the contribution of the spurious background at incredibly low levels.

The technique proposed in this paper is the combination in a single device of all the demanding features needed by next generation experiments: high energy resolution, low cost mass scalability, flexibility in the choice of the double beta decaying isotope, and many powerful handles to keep the background negligible. Its novelty is the enhancement and optimization of the collection of the scintillation light emitted by ultra-pure crystals through the use of arrays of high performance silicon photodetectors cooled to $120 \mathrm{~K}$. This would provide scintillation detectors with $1 \%$ level energy resolution and a $\mu$ s time resolution. We show that with a small array ( $\sim 54 \mathrm{~kg}$ ) of such detectors it is possible to yield in only 1 year of measurement a competitive physics result in the search for the neutrinoless double beta decay of ${ }^{100}$ Mo using natural $\mathrm{CaMoO}_{4}$ scintillating crystals (i.e. without enriching in ${ }^{100} \mathrm{Mo}$ or depleting in ${ }^{48} \mathrm{Ca}$ isotopes). This result would pave the way to a new class of ton scale experiments to search not only for neutrinoless double beta decay with potentially zero background, but also for dark matter and neutrino charged current reactions. 


\section{Neutrinoless double beta decay}

In recent years, the discovery of neutrino oscillations unambiguously proved that neutrinos are massive particles and thus provided the first confirmation of the existence of new physics beyond the standard model [1]. However oscillation experiments cannot shed light on the fundamental open issues concerning the absolute mass values or the quantum nature (Dirac or Majorana fermions) of neutrinos. Neutrinoless double beta decay is a lepton number violating process with the unique feature of being the only feasible means to provide insight into both the above questions. In fact its existence would imply that neutrinos are massive Majorana fermions and could put important constraints on the absolute mass scale $[2,3]$.

Double beta decay $(\beta \beta)$ is a very rare nuclear process in which a nucleus $(\mathrm{A}, \mathrm{Z})$ decays into its $(\mathrm{A}, \mathrm{Z}+2)$ isobar. As the expected decay rates are extremely low, the choice of the parent nuclei is limited to those which are more bounded than the intermediate ones (to avoid the background caused by the sequence of two single beta decays): this condition is met for a number of even-even nuclei. In the Standard Model of Particle Physics $\beta \beta$ is allowed with the contemporary emission of 2 electrons and 2 anti-neutrinos $(\beta \beta 2 v)$, and it has indeed been observed experimentally in a dozen of isotopes with half-lives of the order $10^{18}-10^{21}$ years. Non-standard decay channels appear whenever the Majorana character of the neutrino is allowed. In this case the lepton number is not conserved and neutrinoless decay modes are possible. Neutrinoless double beta decay $(\beta \beta 0 v)$ can proceed via different mechanisms, the simplest one being the virtual exchange of a light Majorana neutrino between the two nucleons. In this case the decay rate is proportional to the square of the socalled effective Majorana mass $\left|\left\langle m_{\beta \beta}\right\rangle\right|$ :

$\left[T_{1 / 2}^{0 \nu}\right]^{-1}=\frac{\left|\left\langle m_{\beta \beta}\right\rangle\right|^{2}}{m_{e}^{2}} G^{0 \nu}\left|M^{0 \nu}\right|^{2}$

where $T_{1 / 2}^{0 v}$ is the decay half-life, $G^{0 v}$ is the two-body phase-space integral, $M^{0 v}$ is the $\beta \beta 0 v$ nuclear matrix element (NME), and $m_{e}$ is the electron mass. The product $F_{N}^{0 v}=G^{0 v}\left|M^{0 v}\right|^{2}$ includes all the nuclear details of the decay and it is usually referred to as nuclear factor of merit. While $G^{0 v}$ can be calculated with reasonable accuracy, the NME value is strongly dependent on the nuclear model used for its evaluation. Significant improvements towards compatibility among various theoretical models have been achieved in recent years, even if discrepancies of about a factor 2-3 still exist. Figure 1 summarizes the current situation [4-13].

The effective Majorana mass is a coherent sum over mass eigenstates with complex Majorana phases and can be parametrized as a function of neutrino oscillation parame-

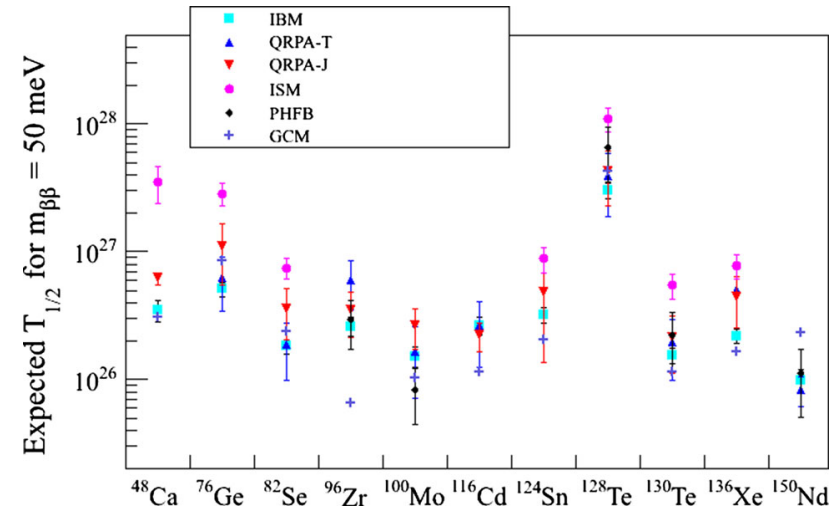

Fig. 1 Neutrinoless double beta decay half-lives calculated for $\left|\left\langle m_{\beta \beta}\right\rangle\right|=50 \mathrm{meV}$ with different most recent theoretical models for several $\beta \beta 0 v$ candidates

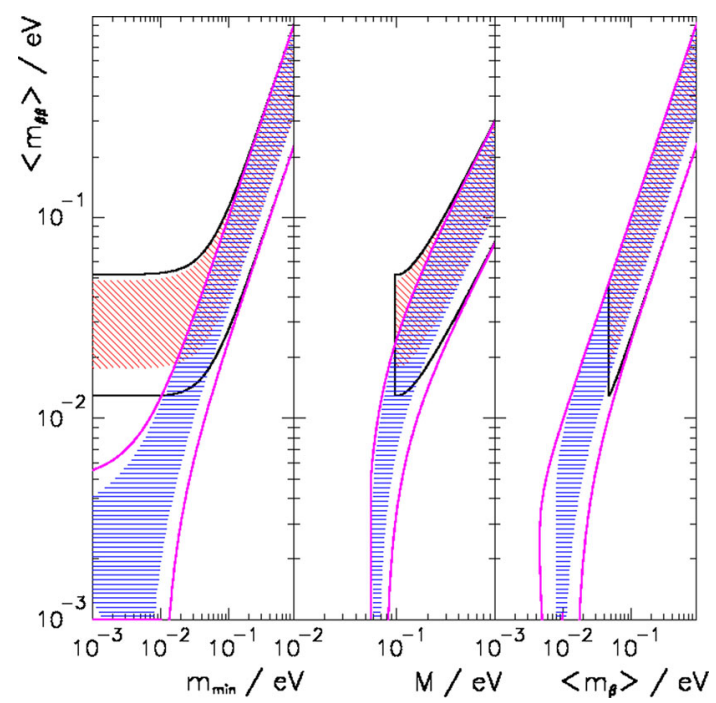

Fig. 2 The left panel shows the expected ranges for $\left|\left\langle m_{\beta \beta}\right\rangle\right|$ as a function of the lightest neutrino mass. The two sets of solid lines correspond to the normal (lighter line with horizontal hatch) and inverted (darker line with diagonal hatch) Hierarchies, which merge for $\left|\left\langle m_{\beta \beta}\right\rangle\right| \geq 0.1 \mathrm{eV}$ into the degenerate mass pattern. The width of the hatched areas is due to the unknown Majorana phases and thus irreducible. The central and right panels show the dependencies of $\left|\left\langle m_{\beta \beta}\right\rangle\right|$ as a function of the summed neutrino mass and of the kinematical mass $\left|\left\langle m_{\beta}\right\rangle\right|$ (reproduced from [1])

ters. The expected allowed ranges for $\left|\left\langle m_{\beta \beta}\right\rangle\right|$ as a function of the lightest neutrino mass are depicted in Fig. 2, where the solid lines are obtained by taking into account the errors of the oscillation parameters (at $3 \sigma$ level).

Experimentally, $\beta \beta 0 v$ searches rely on the measurement of the two electron signal. In the so-called homogeneous or calorimetric experiments the active volume of the detector contains the double beta decaying isotope ( source $=$ detector) . Since the energy of the recoiling nucleus is negligible, the sum kinetic energy of the two electrons is equal to the Qvalue of the transition $\left(Q_{\beta \beta}\right)$. This monochromatic signal, is the main signature used by experiments. On the other hand, 
given the rarity of the process, the gathering of the $\beta \beta 0 v$ counts is hindered by the background events in the energy region under investigation. The sensitivity of a given $\beta \beta 0 v$ experiment is usually expressed in term of the detector factor of merit $F_{D}^{0 v}$, defined as the process half-life corresponding to the maximum signal that could be hidden by a background fluctuation $n_{B}$ at a given statistical confidence level (CL). At $1 \sigma$ level this is given by:

$F_{D}^{0 v}=T_{1 / 2}^{\text {Back.Fluct }}=\ln 2 N_{\beta \beta} \frac{T}{n_{B}}=\ln 2 \frac{x \eta \epsilon N_{A}}{A} \sqrt{\frac{M T}{B \Delta}} f(\Delta)$

with $n_{B}=\sqrt{B T M \Delta}$, where $B$ is the background level per unit mass, energy, and time, $M$ is the detector mass, $T$ is the measuring time, $\Delta$ is the FWHM energy resolution, $N_{\beta \beta}$ is the number of $\beta \beta$ decaying nuclei under observation, $x$ is the stoichiometric multiplicity of the element containing the $\beta \beta$ candidate, $\eta$ is the $\beta \beta$ candidate isotopic abundance, $N_{A}$ is the Avogadro number, $A$ is the compound molecular mass, and $\epsilon$ is the detection efficiency. Finally, $f(\Delta)$ is the "analysis efficiency", i.e. the fraction of signal events that fall in an energy window equal to the FWHM $\Delta$ centered on $Q_{\beta}$ (region of interest or ROI). The experimental sensitivity $F_{D}^{0 v}$ is then translated into an effective Majorana mass sensitivity as $\left|\left\langle m_{\beta \beta}\right\rangle\right|^{-1}=\sqrt{F_{D}^{0 v} \cdot F_{N}^{0 v}}$ showing both the slow dependence (only with the fourth root) of $\left|\left\langle m_{\beta \beta}\right\rangle\right|$ on the crucial experimental parameters and how the isotope choice plays a role in the game from the nuclear model point of view.

Apart from one controversial claim of evidence (in tension with several recent experimental results [14-16]), no $\beta \beta 0 v$ signal has been detected so far. Experimental half-life lower limits exist for many isotopes. Current best sensitivities have been obtained for ${ }^{76} \mathrm{Ge},{ }^{82} \mathrm{Se},{ }^{100} \mathrm{Mo},{ }^{130} \mathrm{Te}$, and ${ }^{136} \mathrm{Xe}$, and are at the level of $10^{24}-10^{25}$ years, which translate to $\left|\left\langle m_{\beta \beta}\right\rangle\right|$

Table 1 Properties of several $\beta \beta 0 v$ candidates: $Q$-value, isotopic abundance, $\beta \beta 2 v$ half-life. $R_{2 v}$ is the rate due to $\beta \beta 2 v$ in a FWHM of $50 \mathrm{keV}$ centered around $Q_{\beta \beta}$ and is calculated using the Primakoff and Rosen approximation [24]. $R_{0 v}$ is the range of $\beta \beta 0 v$ count rates (from the

\begin{tabular}{llllllr}
\hline Isotope & $\begin{array}{l}Q_{\beta \beta} \\
{[\mathrm{keV}]}\end{array}$ & $\begin{array}{l}\eta \\
{[\%]}\end{array}$ & $\begin{array}{l}T_{1 / 2}^{2 v} \\
{[\mathrm{y}]}\end{array}$ & $\begin{array}{l}R_{2 v}(50 \mathrm{keV}) \\
{[\mathrm{c} / \mathrm{y} / \text { ton_iso }]}\end{array}$ & $\begin{array}{l}R_{0 v}\left(m_{\beta \beta}=50 \mathrm{meV}\right) \\
{[\mathrm{c} / \mathrm{y} / \mathrm{ton} \text { iso }]}\end{array}$ & $\begin{array}{l}R_{0 v}\left(m_{\beta \beta}=10 \mathrm{meV}\right) \\
{[\mathrm{c} / \mathrm{y} / \mathrm{ton} \text { iso }]}\end{array}$ \\
\hline${ }^{48} \mathrm{Ca}$ & 4274 & 0.2 & $4.4 \times 10^{19}$ & $4.0 \times 10^{-2}$ & $1.4-23.1$ & $0.1-0.9$ \\
${ }^{76} \mathrm{Ge}$ & 2039 & 7.6 & $1.8 \times 10^{21}$ & $2.5 \times 10^{-2}$ & $1.2-12.2$ & $0.05-0.5$ \\
${ }^{82} \mathrm{Se}$ & 2996 & 8.7 & $9.2 \times 10^{19}$ & $6.8 \times 10^{-2}$ & $4.4-38.7$ & $0.2-1.5$ \\
${ }^{96} \mathrm{Zr}$ & 3348 & 2.8 & $2.3 \times 10^{19}$ & $1.3 \times 10^{-1}$ & $3.9-50.7$ & $0.2-2.0$ \\
${ }^{100} \mathrm{Mo}$ & 3034 & 9.6 & $7.1 \times 10^{18}$ & $6.8 \times 10^{-1}$ & $8.9-71.3$ & $0.4-2.9$ \\
${ }^{116} \mathrm{Cd}$ & 2814 & 7.5 & $2.8 \times 10^{19}$ & $2.1 \times 10^{-1}$ & $6.8-23.8$ & $0.3-1.0$ \\
${ }^{130} \mathrm{Te}$ & 2528 & 34.2 & $6.8 \times 10^{20}$ & $1.3 \times 10^{-2}$ & $3.6-24.9$ & $0.1-1.0$ \\
${ }^{136} \mathrm{Xe}$ & 2458 & 8.9 & $2.1 \times 10^{21}$ & $4.8 \times 10^{-3}$ & $2.5-14.0$ & $0.1-0.6$ \\
${ }^{150} \mathrm{Nd}$ & 3368 & 5.6 & $8.2 \times 10^{18}$ & $2.3 \times 10^{-1}$ & $9.1-42.7$ & $0.4-1.7$
\end{tabular}

values between 0.2 and $0.7 \mathrm{eV}$, depending on the $\beta \beta 0 v$ candidate and on the corresponding NME (see [17] for a recent review).

To improve these results several experimental programs are currently on the way with a variety of challenging techniques and with the common goal of approaching the inverted hierarchy (IH) region of the mass spectrum (see Fig. 2) in the next years. They require not only very strong efforts towards the reduction of the radioactive background in the region of interest, below the intrinsic limit set by the $\beta \beta 2 v$ continuous spectrum, but also good energy resolutions (approximately better than $2 \%$ at $Q_{\beta \beta}$ [17]). In fact, an approximate expression for the $\beta \beta 0 v$ signal $S$ to $\beta \beta 2 v$ background $B$ ratio in the ROI can be written as [2]:

$\frac{S}{B}=\frac{m_{e} T_{1 / 2}^{2 v}}{7 R^{6} Q_{\beta \beta} T_{1 / 2}^{0 \nu}}$

where $R=\Delta / Q_{\beta \beta}$ is the fractional FWHM resolution. It is then clear that a bad energy resolution can spoil all background reduction efforts, because of the broadening of the upper tail of the $\beta \beta 2 v$ continuous spectrum. Table 1 reports the predicted count rates due to $\beta \beta 2 v$ in a ROI of $50 \mathrm{keV}$ (therefore assuming a resolution of $1.7 \%$ at $3 \mathrm{MeV}$ ) as well as the number of expected $\beta \beta 0 v$ signal events for two different values of $\left|\left\langle m_{\beta \beta}\right\rangle\right|$ (50 and $10 \mathrm{meV}, 3 \sigma$ boundaries of the IH region): the signal $S$ to background $B$ ratio is safely bigger than one for all candidate isotopes. Moreover, energy resolution is extremely important to correctly model the different background sources contributing to the measured spectra in the energy region of interest. Finally, energy resolution plays a unique role also when discussing the discovery potential of any given experimental approach, since it is a crucial parameter to single-out the $\beta \beta 0 v$ peak over an almost flat background.

different NME values of Fig. 1) expected for two values of $\left|\left\langle m_{\beta \beta}\right\rangle\right|$ (the effect of the analysis efficiency $f(\Delta)$ has been included). Both $R_{2 v}$ and $R_{0 v}$ are expressed in counts per year per ton of $\beta \beta 0 v$ candidate isotope 
Table 2 Main properties of most common calorimetric experimental techniques for $\beta \beta 0 v$ searches. We would like to stress here that the actual performance of a $\beta \beta 0 v$ experiment must take into account also the candidate isotope properties listed in Table 1

\begin{tabular}{lllll}
\hline Experimental approach & $\begin{array}{l}\text { FWHM resolution } \\
<2 \% \text { in the ROI }\end{array}$ & $\begin{array}{l}\text { Mass } \\
\text { scalability }\end{array}$ & $\begin{array}{l}\beta \beta 0 v \text { isotope } \\
\text { flexibility }\end{array}$ & Background reduction \\
\hline Ge diodes & $\mathrm{X}$ & & $\mathrm{X}$ & $\gamma$ vs. $\alpha / \beta$ (event topology by PSD) \\
Bolometers & $\mathrm{X}$ & $\mathrm{X}$ & $\boldsymbol{X} / \gamma$ vs. $\alpha$ (PSD and/or light/heat) \\
Organic scintillators & & $\mathrm{X}$ & & $\beta / \gamma$ vs. $\alpha$ (light/charge), $\gamma$ vs. $\beta$ (topology) \\
Liquid noble gas TPC & & & $\beta \beta$ vs. $\alpha / \beta / \gamma$ (event topology) \\
Noble gas TPC & $\mathrm{X}$ & $\mathrm{X}$ & $\mathrm{X}$ & $\beta / \gamma$ vs. $\alpha$ (PSD), $\gamma$ vs. $\alpha / \beta$ (topology) \\
Inorganic scintillators & $\mathrm{X}$ & & &
\end{tabular}

When the background rate is so low that the expected number of spurious events in the region of interest is close to zero ( $Z B$-zero background condition), the detector factor of merit becomes:

$F_{Z B}^{0 v}=\ln 2 N_{\beta \beta} \frac{T}{n_{L}}=\ln 2 \frac{x \eta \epsilon N_{A}}{A} \frac{M T}{n_{L}} f(\Delta)$

where the background is now described by $n_{L}$, a constant term which represents the maximum number of counts compatible, at a given confidence level, with no counts observed [1]. In this case the sensitivity to the $\beta \beta 0 v$ signal goes linearly not only with the isotopic fraction and the detection efficiency, but also with the detector mass and the measuring time, a particularly appealing regime for next generation experiments.

From the above discussion the strategy for future experimental programs aiming at exploring the IH region is clear:

- Minimization of the environmental and cosmogenic radioactive background (experiments placed underground with heavy shielding, careful selection of radiopure materials to build the experimental set-up, detector technology with active event identification capability and/or event position reconstruction);

- Large detector mass, on the tonne scale or larger, and mass scalability;

- A well performing detector (good energy resolution and time stability), with flexibility in the choice of the $\beta \beta 0 v$ isotope;

- A favourable $\beta \beta 0 v$ candidate isotope (high $Q_{\beta \beta}$ value, high natural isotopic abundance, short half-life, large ratio of $\beta \beta 0 v / \beta \beta 2 v$ in the ROI)

- A reliable and easy to operate detector technology requiring little service on long run times;

Any of the proposed new projects [17] is a compromise among these often conflicting requests (see also Table 2 ). We show that large arrays of inorganic scintillators could address all the above items in a single approach.

\section{The benefit of scintillation detectors}

In realm of $\beta \beta 0 v$ calorimetric experiments, many techniques are available. So far Ge diode detectors and bolometers have provided excellent energy resolutions [18-21], at the level of few parts per thousand, and both offer promising solutions for background reduction. On the other hand, both techniques suffer from limitations in the achievable mass increase (mainly for cost reasons in the case of Ge diodes, and also technological issues in the case of bolometers). On the contrary, large scale organic scintillation detectors provide mass scalability in low-background environment without apparent limitations, but suffer from a seemingly irreducible restriction in the attainable energy resolution [15,22]. Finally, Xe (liquid or gas) based TPC [14,23] are bound to a trade off between source mass and energy resolution, although they may implement powerful, but complex, background abatement concepts. The possibility to implement in a single approach all the critical features - high energy resolution, background abatement techniques and mass scalability - is the true challenge for next generation projects. Indeed, large arrays of inorganic scintillators may be the solution to that.

Inorganic scintillating crystals can be grown with high level of intrinsic radiopurity and good scintillation properties (required for high energy resolution). Moreover, there exist many scintillators containing $\beta \beta 0 v$ candidate nuclei, thus allowing a high flexibility in the choice of the scintillation detector (i.e. a wide range of $\beta \beta 0 v$ candidates) without the limitation imposed by other experimental techniques. Furthermore, thanks to Quenching Factors ${ }^{1}(\mathrm{QF})$ for $\alpha$ particles as small as 0.2 - which practically remove all $\alpha$ background from the energy region around $Q_{\beta \beta}$ - and intrinsic $\alpha / \beta$ discrimination ability (Pulse Shape Discrimination or PSD) useful to tag $\alpha-\beta$ coincidences - scintillators offer powerful tools for background minimization. Finally, large arrays of scintillating crystals can be assembled with quite simple technological skills and in rather low-cost experimental setups.

\footnotetext{
1 The quenching factor is here defined as the ratio of the light yield for an alpha particle or a nuclear recoil to that of an electron or a gamma.
} 
Despite all those features, this approach has not exploited its full potential so far, the main reason being the relatively poor energy resolution achievable with scintillating crystals optically coupled to photomultiplier tubes (see next Sect. 4).

We discuss in this paper an innovative method to read the scintillation light emitted by large mass scintillators containing the $\beta \beta 0 v$ candidate nuclei without degrading the energy resolution. In fact, the limit imposed to the scintillator resolution by the carrier statistics alone is not so crucial. Assuming to have a crystal that emits 10,000 photons per $\mathrm{MeV}$ - a value definitely reasonable for many scintillators [25] - the relative FWHM resolution $R_{\mathrm{Stat}}$ at an energy of $3 \mathrm{MeV}$ (close to the $Q_{\beta \beta}$ of many isotopes) in the hypothesis of being able to collect all the photons can be estimated as $R_{\text {Stat }}(\mathrm{FWHM})=2.355 / \sqrt{3 \cdot 10000}=1.36 \%$, which corresponds to a FWHM energy resolution $\Delta$ of $41 \mathrm{keV}$ at $3 \mathrm{MeV}$. Even if this value is not comparable to the resolution of Ge diodes or bolometers (which is of the order of 4-6 $\mathrm{keV}$ at $3 \mathrm{MeV}$ ), it is not a limiting factor in the case of a low background $\beta \beta 0 v$ experiment (see also Table 1). In the following we will demonstrate that it is possible to maintain this resolution performance even with the complete readout chain. The great advantage of this new method is the availability of a homogeneous detector with the strongest potentialities towards background reduction and the possibility to easily scale the experimental mass to boost the sensitivity towards unexplored regions of the neutrino mass spectrum. This detection concept is compared to the other techniques in Table 2.

\section{The measurement technique}

The basic idea of the proposed new technique is to optically couple suitable scintillating crystals to silicon drift detectors (SDD) at a working temperature around $120 \mathrm{~K}$. Indeed, many scintillating crystals have been used at low temperatures showing improved light emission [26] with respect to room temperature. On the other hand, in the recent years there have been many advances in the light detection using solid state devices [27]. The innovative idea is to combine these features to provide the winning technology for future experiments.

Table 3 lists a selection of scintillating crystals containing interesting $\beta \beta 0 \mathrm{v}$ candidate nuclei with transition energy above the ${ }^{208} \mathrm{Tl} \gamma$ peak (the most energetic and intense $\gamma$ line from natural radioactivity) - crucial issue to fulfill the $\mathrm{ZB}$ condition. All of them have fairly well known scintillation properties at low temperatures $[25,26]$ and offer light yields (LYs) well above the $10000 \mathrm{ph} / \mathrm{MeV}$ value considered in the previous section. Therefore, all of them are potentially good candidates for high resolution detectors. It is worth stressing here that the development of inorganic scintillators is a field
Table 3 A selection of $\beta \beta 0 v$ candidates contained in available scintillating crystals. The range of expected half-lives for an effective mass $\left|\left\langle m_{\beta \beta}\right\rangle\right|$ of $10 \mathrm{meV}$ is reported

\begin{tabular}{lll}
\hline Isotope & $\begin{array}{l}T_{1 / 2}^{0 v}(10 \mathrm{meV}) \\
{\left[10^{27} \text { years }\right]}\end{array}$ & $\begin{array}{l}\text { Available } \\
\text { scintillating crystals }\end{array}$ \\
\hline${ }^{48} \mathrm{Ca}$ & $7-100$ & $\mathrm{CaF}_{2}, \mathrm{CaWO}_{4}, \mathrm{CaMoO}_{4}$ \\
${ }^{100} \mathrm{Mo}$ & $1-9$ & $\mathrm{CaMoO}_{4}, \mathrm{ZnMoO}_{4}$ \\
${ }^{116} \mathrm{Cd}$ & $3-10$ & $\mathrm{CdWO}_{4}, \mathrm{CdMoO}_{4}$ \\
\hline
\end{tabular}

of continuous innovation that keeps on offering new crystals with competitive characteristics for many applications - see e.g. [25].

The key point is to avoid a degradation of the resolution (i.e. a loss of carriers) when the crystal is coupled to a proper device to detect the scintillation signal. For example, the use of commercial phototubes introduces strong limitations because of low quantum efficiency $\epsilon_{Q}$ - of the order of $40 \%$ in the best cases - and rather narrow spectral response, which implies that many of the crystals of Table 3 do not emit light within the wavelength intervals required to match the phototube characteristics, thus causing a further deterioration of the energy resolution. Moreover it is very difficult to obtain commercial phototubes with a low intrinsic radioactivity of the glass envelope. The use of SDDs as photodetectors represents therefore an appealing possibility, since these devices are characterized by a $\epsilon_{Q}$ larger than $80 \%$ in a wide range of wavelenghts [27]. In addition, silicon is one of the cleanest materials from the radioactivity point of view.

To evaluate the energy resolution potentially achievable by coupling SDDs to scintillating crystals, we must consider the additional contribution of the noise of the measurement chain:

$$
\begin{aligned}
R(\mathrm{FWHM}) & =\sqrt{R_{\text {stat }}^{2}+R_{\text {noise }}^{2}} \\
& =2.355 \sqrt{\frac{1}{\epsilon_{Q} \cdot N_{p h}}+\frac{\mathrm{ENC}_{e}^{2}}{\epsilon_{Q}^{2} \cdot N_{p h}^{2}}}
\end{aligned}
$$

where $\mathrm{ENC}_{e}$ is the equivalent noise charge of the electronic chain and $N_{p h}$ is total number of photons emitted by the scintillating crystal after an energy deposition. A competitive detector therefore requires to reduce as much as possible the term $R_{\text {noise }}$. In practice, one should keep this term at least within a tenth of the statistical one: $\mathrm{ENC}_{\mathrm{e}}=$ $\sqrt{\epsilon_{Q} \cdot N_{p h}} / 10=\sqrt{0.8 \cdot 30000} / 10=16 e^{-}$rms, where a $\mathrm{LY}$ of $10,000 \mathrm{ph} / \mathrm{MeV}$ at an energy of $3 \mathrm{MeV}$ has been considered. This value of $\mathrm{ENC}_{e}$ is quite demanding but is within reach of presently available technology, as we will discuss in the following Sect. 4.1.

Another possible approach that can be adopted to readout the signal emitted by the scintillating crystals is the use of a different solid state device: the silicon photomultiplier 
(SiPM). The optimization of this photodetector had a strong development in the last few years. The noise induced by such type of device operated at low temperatures is negligible; on the other hand the main limitations, at the moment, are given by the lower quantum efficiency with respect to the SDDs (due to the larger dead layers that are actually needed to realize a "large" surface detector) and by the strong non linearity that can be observed when measuring a large number of photons (due to the intrinsic avalanche signal production in a single cell). Since the optimization of SiPM is expected to continue in the future, it is not excluded that also these devices will became suitable for a rare event search with the same performances that we point out in this paper for the SDDs.

\subsection{Silicon drift detectors as photodetectors}

The keystone for the success of the proposed technique is the achievable noise performance of the SDD detectors. Considering a solid state device with capacity $C_{d}$ coupled to a charge preamplifier with input capacitance $C_{i}$ and series noise $\left\langle e_{w}^{2}\right\rangle$, the equivalent noise charge can be written as [28]:

$$
\begin{aligned}
\mathrm{ENC}= & {\left[\frac{k_{1} \cdot\left\langle e_{w}^{2}\right\rangle \cdot\left(C_{d}+C_{i}+C_{p}\right)^{2}}{\tau}\right.} \\
& \left.+k_{3} A_{1 / f}\left(C_{d}+C_{i}+C_{p}\right)^{2}+2 k_{2} q I_{l} \tau\right]^{1 / 2}
\end{aligned}
$$

where $C_{p}$ is the parasitic capacitance between the detector and the amplifying circuit, $I_{l}$ is the leakage current both at the input of the gate of the FET preamplifier and of the solid state detector, $q$ is the electron charge $\left(1.6 \times 10^{-19} \mathrm{C}\right)$, and $\tau$ is the shaping time of the acquired signal. $k_{1}, k_{2}$ and $k_{3}$ are parameters related to the type of shaping form used in the electronic chain (Gaussian, triangular, trapezoidal, etc.) - at this level all of them can be assumed of unit value, also in view of a possible application of an optimum filter procedure [28] as it is actually done in many experiments. The three terms in the ENC expression are the so called series noise, l/f noise, and parallel noise, respectively. As the above formula shows, the achievement of low levels of ENC requires a careful design and selection of all components: the detector, the amplifier front-end, and the coupling between the two. In particular:

- The contribution of the $1 / \mathrm{f}$ noise can be kept at negligible levels compared to the other terms through a proper choice of the input device for the preamplifier (for example, a JFET) [29].

- SDDs are characterized by a very low capacitance $C_{d}$ of the electrode collecting the signal charge [30], typically of the order of $0.5-1 \mathrm{pF} / \mathrm{cm}^{2}$; moreover, the front-end electronics can be integrated directly on the same wafer of the photodetector [31], thus minimizing $C_{p}$.
- The leakage current is one of the most critical parameters. Its value is related to the size of the solid state detector and is approximatly proportional to the area of the detector. Typical values at room temperature are of the order of $1 \mathrm{nA} / \mathrm{cm}^{2}$ for many silicon devices. Since a desirable order of magnitude for the detector surface - to collect the light from a scintillating crystal devoted to $\beta \beta 0 v$ search - is of tens of square centimeters, this would result in a leakage current of several nA that would spoil the ENC. On the other hand, measurements on SDDs [27] show a marked decrease in the leakage current with decreasing temperature. In principle this decrease should have an exponential trend: therefore the leakage current could become negligible by reducing the operating temperature, although carrier freeze-out sets a lower limit around $77 \mathrm{~K}$ [32]. An extrapolation below $-25^{\circ} \mathrm{C}$ of the experimentally observed behaviour of $I_{l}$ in SDDs shows that a working temperature around $120 \mathrm{~K}$ should be enough to have a leakage current as low as $10^{-14} \mathrm{~A}$ per $\mathrm{cm}^{2}$, value observed for many semiconductor devices at this temperature. Moreover, at low temperatures also the leakage current of the input JFET of the preamplifier becomes negligible: therefore it is advantageous to place this component at the cold stage of the experimental set-up too. Another advantage of placing both the detector and the preamplifier in close vicinity low temperatures is that the parasitic capacitance $C_{p}$ can be reduced to small values $\left(C_{p} \sim 0.5 \mathrm{pF}\right)$ therefore diminishing its contribution to the first two terms of ENC.

- The series noise of the preamplifier is given by: $\left\langle e_{w}^{2}\right\rangle=$ $2 k_{B} T \alpha / g_{m}$ where $k_{B}$ is the Boltzmann constant, $T$ is the operating temperature, $g_{m}$ is the transconductance of the input JFET, and $\alpha=0.7$ for an ideal JFET. The value of the transconductance increases with the JFET area, therefore by increasing the size of the device it is possible to achieve a sensible reduction of $\left\langle e_{w}^{2}\right\rangle$. On the other hand this would cause an increase of both the leakage current and of the input capacitance $C_{i}$. Also in this case a low operating temperature [28] helps the optimization of the device geometry keeping a small area to reduce $I_{l}$ and $C_{i}$ while controlling $\left\langle e_{w}^{2}\right\rangle$ with a proper choice of the working temperature, thus achieving a reduction of the total ENC. Typical values of $\left\langle e_{w}^{2}\right\rangle$ at temperatures of about $120-150 \mathrm{~K}$ are of the order of $10^{-18} \mathrm{~V}^{2} / \mathrm{Hz}$ while for the input capacitance we can assume $C_{i} \sim 1 \mathrm{pF}$.

In conclusion, an SDD of $1 \mathrm{~cm}^{2}$ operated at a temperature of about $120-150 \mathrm{~K}$ and coupled to a JFET closely placed are a viable solution for reading out the light emitted by a scintillating crystal with high resolution. To evaluate the expected total noise $\mathrm{ENC}_{e}$ associated to such an electronic chain we can choose a shaping time of about $50 \mu \mathrm{s}$, an acceptable value for the typical decay times of inorganic scintillators, 
Table 4 Properties of $\mathrm{CaMoO}_{4}, \mathrm{CdWO}_{4}$, and $\mathrm{CaF}_{2}(\mathrm{Eu})$ scintillating crystals. The values at low temperatures are extrapolated from the cited references. The absorption length is an average value of the many different data reported in the literature

\begin{tabular}{|c|c|c|c|c|c|c|}
\hline \multirow[t]{2}{*}{ Property } & \multicolumn{2}{|l|}{$\mathrm{CaMoO}_{4}$} & \multicolumn{2}{|l|}{$\mathrm{CdWO}_{4}$} & \multicolumn{2}{|l|}{$\mathrm{CaF}_{2}(\mathrm{Eu})$} \\
\hline & $300 \mathrm{~K}$ & $120 \mathrm{~K}$ & $300 \mathrm{~K}$ & $120 \mathrm{~K}$ & $300 \mathrm{~K}$ & $200 \mathrm{~K}$ \\
\hline Atomic mass $[\mathrm{g} / \mathrm{mol}]$ & 200 & & 360 & & 78 & \\
\hline Density $\left[\mathrm{g} / \mathrm{cm}^{3}\right]$ & $\sim 4.3[42]$ & & 7.9 [37] & & $3.2[26]$ & \\
\hline Melting point $\left[{ }^{\circ} \mathrm{C}\right]$ & $\sim 1445$ [42] & & $1271[37]$ & & $1418[38]$ & \\
\hline Lattice structure & Scheelite & & Wolframite & & Fluorite & \\
\hline Energy gap $E_{g}[\mathrm{eV}]$ & $4.0[26]$ & & $4.2[26]$ & & & \\
\hline Emission maximum $\lambda_{\max }[\mathrm{nm}]$ & $520[42]$ & $\sim 530[36]$ & $480[37]$ & $480[26]$ & $435[38]$ & \\
\hline Light yield $[\mathrm{ph} / \mathrm{MeV}]$ & $\sim 8900[36]$ & $\sim 25000[36]$ & $\sim 18500[26]$ & $\sim 33500[26]$ & $24000[39]$ & $\sim 26400[40]$ \\
\hline Scintillation decay time $[\mu \mathrm{s}]$ & $\sim 18[36]$ & $\sim 190[36]$ & $13[37]$ & $\sim 22[26]$ & $0.9[39]$ & \\
\hline Refractive index & $1.98[36]$ & & $2.2-2.3[37]$ & & $1.44[39]$ & \\
\hline Absorption length [cm] & $\sim 60$ & & $\sim 60$ & & & \\
\hline
\end{tabular}

especially at low temperatures [26]. With this selection of $\tau$, together with the values of the capacitances, $\left\langle e_{w}^{2}\right\rangle$ and $I_{l}$ just inferred, the contributions of the series and of the parallel noises to the total $\mathrm{ENC}_{e}$ are comparable:

$$
\begin{aligned}
\mathrm{ENC}_{\mathrm{e}} & =\frac{\mathrm{ENC}}{1.6 \times 10^{-19}}=\frac{\sqrt{8 \times 10^{-38}+1.6 \times 10^{-37}}}{1.6 \times 10^{-19}} \\
& =3.1 \mathrm{e}^{-} \mathrm{rms}
\end{aligned}
$$

This level of noise is for one solid state photodetector of $1 \mathrm{~cm}^{2}$ of total area. On the other hand, for applications which need to cover large areas with light sensitive detectors - like the one proposed in this paper - bigger size SDDs would be necessary. A possible solution - which may have additional advantages for background reduction, as discussed later - is to use several independent small area SDDs to cover completely the surface of interest. The use of SDDs for segmented readout has already given interesting results for $\gamma$ and X-ray detection [33-35]. A segmented optical readout has the additional benefit of keeping small drift times within each SDD, i.e. the time needed by the charge generated inside the SDD by an optical photon to reach the collecting anode. This parameter influences mainly the time response of the SDD. On the other hand, the number of SDDs $\left(N_{\mathrm{SDD}}\right)$ used to readout the emitted scintillation light has to be included in the evaluation of the overall energy resolution: assuming $\sim 40$ SDDs per detector (see later), the additional contribution to the resolution amounts to $\sqrt{N_{\mathrm{SDD}}} \times \mathrm{ENC}_{\mathrm{e}}=\sqrt{40} \times$ $3.1 \sim 19.6 e^{-} r m s$, very close the initial goal of $16 e^{-} r m s$, and looks indeed extremely promising.

\subsection{Scintillating crystal choice}

Among the different scintillating crystals listed in Table 3, $\mathrm{CaMoO}_{4}, \mathrm{CdWO}_{4}$, and $\mathrm{CaF}_{2}$ look very promising to build large scintillation detector arrays devoted to rare event searches. Their main physical properties are listed in Table 4. In order to give relevance to the discussion, in the following we will refer to $\mathrm{CaMoO}_{4}$ crystals, even if it is clear that similar considerations are valid also for $\mathrm{CdWO}_{4}, \mathrm{CaF}_{2}$, and in general for any scintillating crystal with adequate physical properties.

$\mathrm{CaMoO}_{4}$ is an intrinsic scintillator with a high molybdenum content ( $48 \%$ in mass), thus extremely attractive for the search of the $\beta \beta 0 v$ of ${ }^{100} \mathrm{Mo}$, which in turn is one of the most interesting isotopes because of its high transition energy and relatively high natural isotopic abundance (Table 1). Moreover the relatively low $\mathrm{Z}$ of $\mathrm{CaMoO}_{4}$ reduces its efficiency for $\gamma$ detection, with the largest $\gamma$ contribution to counts in the ROI coming from the $3143 \mathrm{keV}{ }^{214} \mathrm{Bi}$ line $\left({ }^{238} \mathrm{U}\right.$ decay chain, branching ratio: $\left.0.0012 \%\right)$. Also the thermal neutron cross section is very low, while the only dangerous cosmogenic background is the $\beta^{+}$decay of ${ }^{88} \mathrm{Y}$ $\left(Q_{\beta+}=3.62 \mathrm{MeV}\right)-$ produced by spallation on ${ }^{100} \mathrm{Mo}-$ which anyway has a short half-life ( $\tau_{1 / 2}=106.6$ days $)$. On the other hand, among the variety of inorganic materials containing $\mathrm{Mo}, \mathrm{CaMoO}_{4}$ shows a relative brighter scintillation at room temperature, which increases with lowering the operating temperature.

Concerning the intrinsic radiopurity level attainable in $\mathrm{CaMoO}_{4}$, recent efforts on the careful check of all raw materials needed for the crystal growing as well as a close control of all crystallization steps have allowed to reach internal concentrations as low as $10 \mu \mathrm{Bq} / \mathrm{kg}$ for both ${ }^{238} \mathrm{U}$ and ${ }^{232} \mathrm{Th}$ [41]. Another important feature of $\mathrm{CaMoO}_{4}$ is a measured QF of 0.2 [42], which prevents the dangerous alpha background from contributing to the ROI. Moreover, $\mathrm{CaMoO}_{4}$ offers a pulse-shape discrimination (PSD) capability between $\gamma / \beta$ events and $\alpha$ particles, which is very useful in the understanding and reduction of background spectra (see later): in [42] the demonstrated discrimination capability was at the 
level of $\sim 90 \%$, but it was recently measured to be higher than $99.9 \%$ [41].

A drawback in the use of natural $\mathrm{CaMoO}_{4}$ as detector for the search of the $\beta \beta 0 v$ of ${ }^{100} \mathrm{Mo}$ is the presence of the isotope ${ }^{48} \mathrm{Ca}$, which is also a $\beta \beta 0 v$ candidate with a high transition energy (see Table 1). Despite its very low isotopic abundance, the expected count rate due to the continuous spectrum of the $\beta \beta 2 v$ of ${ }^{48} \mathrm{Ca}$ in the ROI $(\sim 50 \mathrm{keV})$ of ${ }^{100} \mathrm{Mo}$ is at the level of $\sim 10^{-2}$ counts/(keV $\left.\cdot \mathrm{kg} \cdot \mathrm{y}\right)$. This must be taken into account when conceiving a $\beta \beta 0 v$ experiment aiming at exploring the $\mathrm{IH}$ region of the neutrino mass spectrum. In this respect, recent developments in the production of ${ }^{40} \mathrm{Ca}^{100} \mathrm{MoO}_{4}$ crystals, isotopically depleted in ${ }^{48} \mathrm{Ca}$ and enriched in ${ }^{100} \mathrm{Mo}$, with scintillation properties very similar to those of the natural crystals [43] already offer a viable solution to this problem.

\section{Expected detector performance}

A prototype detector single module to prove this new measuring technique can be conceived as made of a cylindrical scintillating crystal with the two flat circular surfaces optically coupled to two arrays of SDD photodetectors. Scintillating crystal indicative dimensions could be $5 \mathrm{~cm}$ of diameter and $6 \mathrm{~cm}$ of height. We imagine each crystal to be laterally covered with a reflective sheet or paint to improve the light collection, and coupled to the SDD array by means of a suitable optical grease. Given the above crystal dimensions, about 40 exagonal SDDs of $\sim 1 \mathrm{~cm}^{2}$ of area are needed to completely cover the two circular surfaces of the cylinder. ${ }^{2}$

To evaluate the expected energy resolution of a single detector module, besides the contributions of the statistical term and of the electronics noise, it is necessary to take into account also the capability to collect all the generated photons on the entrance window of the solid state photodetector. For this purpose, a dedicated Monte Carlo simulation based on the GEANT4 toolkit [44] was developed with the main goal of understanding the dependence of the photon collection efficiency on the type of coupling to the scintillating crystal. In the simulation, the scintillator is described as a cylindrical crystal of $\mathrm{CaMoO}_{4}$ with the above dimensions and the characteristics listed in Table 4. The lateral crystal surfaces can be chosen to be polished or covered with reflecting materials to vary the probability that a photon is refracted outside the crystal and then lost. On the two circular surfaces of the cylinder it is possible to put a coupling layer of $0.1 \mathrm{~mm}$ of air (refraction index 1.003) or optical grease (refraction index

\footnotetext{
2 The optimization of the number of photodetectors per crystal surface as well as of the area of the single SDD must be addressed on the basis of the measured performance of the solid state detectors.
}

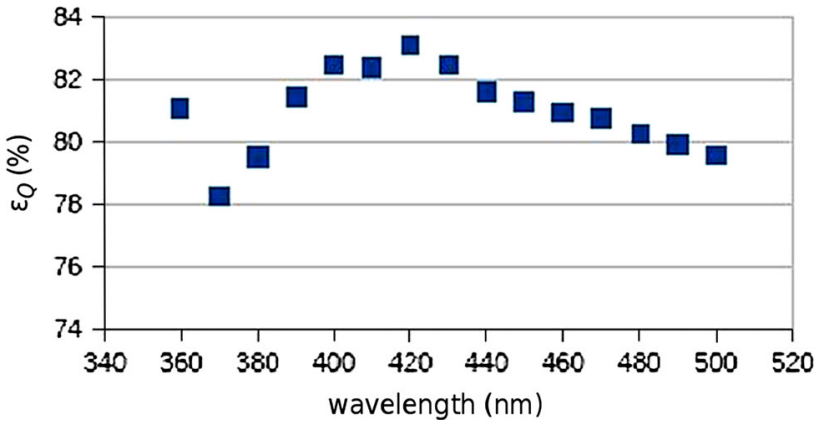

Fig. 3 Quantum efficiency reconstruction from [27] for a SDD solid state detector

1.6) and, additionaly, a SDD detector with a refraction index of 1.45 and the $\epsilon_{Q}$ shown in Fig. 3.

The preliminary simulation results are reported in Table 5 . They show that the fraction of collected light is strongly enhanced by the presence of a grease interface between the crystal and the silicon device and by the covering of the lateral crystal surfaces with a diffused reflector. The fraction of photons generated inside the $\mathrm{CaMoO}_{4}$ crystal which reach the SDD photodetector seems very close to $60 \%$ (including the SDD $\epsilon_{Q}$ ), roughly confirming data available in the literature [45]. These results can be further optimized by using a more precise design of the photodetectors, of the optical coupling to the scintillator, and of the crystal lateral surface treatment.

Finally, the FWHM resolution attainable at an energy of $3 \mathrm{MeV}$ with the conceived single detector module operated at $120 \mathrm{~K}$ can be evaluated as:

$$
\begin{aligned}
& R(\mathrm{FWHM})=\sqrt{R_{\text {int }}^{2}+R_{\mathrm{Stat}}^{2}+R_{\text {noise }}^{2}} \\
& \quad \simeq 2.355 \sqrt{\frac{1}{\alpha_{p h} N_{p h} \epsilon_{Q}}+\frac{N_{\mathrm{SDD}} \mathrm{ENC}_{e}^{2}}{\alpha_{p h}^{2} N_{p h}^{2} \epsilon_{Q}^{2}}}=1.15 \%
\end{aligned}
$$

where $\alpha_{p h} \sim 70 \%$ is the light collection efficiency just discussed, $N_{p h} \sim 75,000$ is the total number of photons generated inside a $\mathrm{CaMoO}_{4}$ crystal operated at $120 \mathrm{~K}$ after an energy deposition of $3 \mathrm{MeV}, \epsilon_{Q} \sim 80 \%$ is the SDD quantum efficiency, and $\sqrt{N_{\mathrm{SDD}}} \times \mathrm{ENC}_{e} \sim 20 e^{-}$rms is the equivalent noise charge value calculated above. In the general expression of the total FWHM resolution it has been introduced also the contribution of $R_{\text {int }}$ which is the intrinsic resolution of the scintillator and is related to several effects, like inhomogeneities due to the local variation of the light output in the scintillating crystal, variations of the reflectivity of the diffuse reflector surrounding the scintillator, as well as nonproportionalities of the scintillator response $[46,47]$. At the energies of interest for $\beta \beta 0 v$ searches $(\sim 3 \mathrm{MeV})$ the contribution of $R_{\text {int }}$ can be completely neglected, but this assumption must be verified at energies lower than $1 \mathrm{MeV}$. Therefore the predicted FWHM resolution at $3 \mathrm{MeV}$ of a $\mathrm{CaMoO}_{4}$ detector coupled to 40 SDDs which read the scintillation light is 
Table 5 Fraction of revealed optical photons depending on the type of crystal lateral surface treatment and on the kind of coupling to the scintillating crystal

\begin{tabular}{llll}
\hline $\begin{array}{l}\mathrm{CaMoO}_{4} \\
\text { lateral surface treatment }\end{array}$ & $\begin{array}{l}\text { Coupling layer: } \\
\text { AIR }\end{array}$ & $\begin{array}{l}\text { Coupling layer: } \\
\text { GREASE }\end{array}$ & $\begin{array}{l}\text { Coupling layer: } \\
\text { GREASE+SDD }\end{array}$ \\
\hline Polished & 0.126 & 0.315 & 0.252 \\
Polished with specular coating & 0.125 & 0.312 & 0.254 \\
Polished with specular wrapping & 0.125 & 0.311 & 0.252 \\
Polished with dffusive coating & 0.361 & 0.692 & 0.562 \\
Polished with dffusive wrapping & 0.126 & 0.312 & 0.255 \\
\hline
\end{tabular}

of about $37 \mathrm{keV}$ : this is a very good value indeed, that allows the minimization of the ${ }^{100} \mathrm{Mo} \beta \beta 2 \nu$ contribution to the ROI as well as a real discrimination capability of the expected $\beta \beta 0 v$ sharp peak over the background events.

The attainable time resolution of such a detector module is directly connected to the scintillation decay time constant and the total photon emission (Hyman theory [48]) through: $t^{2}=2 \tau_{r} \tau_{d} N(t) / N_{\text {phe }}$ where $N(t)$ is the total number of photoelectrons needed to overcome the trigger threshold, $N_{p h e}$ is the total number of photoelectrons collected by the photodetector, $\tau_{d}$ is the scintillator decay time constant, and $\tau_{r}$ is the photodetector rise time. In the case of SDDs, $\tau_{r}$ is the time needed to drift the electrons towards the anode $(\sim 1 \mu \mathrm{s})$. Therefore, using the the data reported in Table 4 for $\mathrm{CaMoO}_{4}$ at a temperature of $120 \mathrm{~K}$, and assuming a threshold six time larger than the evaluated rms noise of $20 \mathrm{e}^{-}$, a time resolution of $\sim 1 \mu \mathrm{s}$ can be expected (for $1 \mathrm{MeV}$ energy deposition), completely dominated by the SDD drift time.

Finally, an additional potentiality of this type of detectors is the possibility of reconstructing the event topology by exploiting the segmentation of the light readout. In principle the photodetector matrix placed on the scintillator circular surfaces should be able to distinguish a single-site event (like the energy deposition by electrons, as in the case of $\beta \beta 0 v$ ) from a multiple-site event (like an energetic $\gamma$ interaction) of similar energy. Preliminary Monte Carlo simulations - which do not include the optical photon propagation yet - showed that among the $\gamma$ interactions of initial energy equal to $3 \mathrm{MeV}$ depositing the whole energy in a single $\mathrm{CaMoO}_{4}$ crystal, only $30 \%$ would be labelled as single-site event if the threshold distance for distinguishing a multiple-site interaction is set equal to $10 \mathrm{~mm}$; this fraction becomes as low as $14 \%$ if the minimum detectable inter-site distance is $5 \mathrm{~mm}$. These results are referred to interactions of single $\gamma \mathrm{s}$ of $3 \mathrm{MeV}$ : the abatement fraction of the multiple-site events may increase when the $3 \mathrm{MeV}$ energy is deposited by coincident $\gamma \mathrm{s}$ (e.g. coming from the ${ }^{232} \mathrm{Th}$ and ${ }^{238} \mathrm{U}$ chains or from showers caused by muons interacting in the external shielding). These are very preliminary remarks: if confirmed they would open new background discrimination opportunities for this type of detectors.

\section{A prototype array of high performance scintillation detectors for $\beta \beta 0 v$ searches}

In this section we investigate the expected performance of an array of $\mathrm{CaMoO}_{4}$ detectors with SDDs' light readout for the search of the $\beta \beta 0 v$ of ${ }^{100} \mathrm{Mo}$. We suppose the full detector to be composed by $108 \mathrm{CaMoO}_{4}$ cylindrical crystals, $5 \mathrm{~cm}$ of diameter and $6 \mathrm{~cm}$ of height, arranged in four layers of 27 single modules for a total mass of $54 \mathrm{~kg}$. We will consider two options: (1) an array of 108 natural $\mathrm{CaMoO}_{4}$ crystals, and (2) an array of $108{ }^{40} \mathrm{Ca}^{\text {nat }} \mathrm{MoO}_{4}$ crystals, isotopically depleted in ${ }^{48} \mathrm{Ca}$. Each scintillator is laterally covered with a reflective sheet or paint to improve the light collection. The two circular surfaces of every cylindric crystal are optically coupled to an array of $\sim 20$ SDD light sensors each. The 27 single detectors of any layer are lying side by side and mechanically supported by a thin copper grid with teflon gaskets. The tentative design is shown in Fig. 4. The full structure can be enclosed in a $5 \mathrm{~cm}$ thick cylindrical copper cryostat of $\sim 45 \mathrm{~cm}$ of diameter and $\sim 50 \mathrm{~cm}$ of height. The $\mathrm{Cu}$ vessel is vacuum tight and the operating temperature of $120 \mathrm{~K}$ can be provided by an external cryocooler thermally coupled to the detector array. This very simple experimental set-up already reflects many of the shrewdnesses required by a careful background control strategy. In fact, the only materials in close contact with the detectors are high-purity copper, teflon, and silicon, besides the wiring. The whole set-up can be surrounded by at least $20 \mathrm{~cm}$ of lead to shield the experiment from the environmental radioactivity. We imagine this experimental set-up to be located at the Laboratori Nazionali del Gran Sasso, Italy, where an average rock overburden of $\sim 3600$ m.w.e. shields the experiments from cosmic rays.

In order to reach a competitive sensitivity in a $\beta \beta 0 v$ search, as already discussed, it is extremely important to remove any spurious source of counts from the ROI. This is a tricky task, which absorbs most efforts of any experimental programs. Main sources of background are: (1) radioactive contaminations of the detector and of the experimental set-up; (2) environmental muons, neutrons and gammas; (3) cosmogenic activation of the detector and set-up materials. The main strategies for background control include a careful selection of the construction materials, special treatments for reducing 


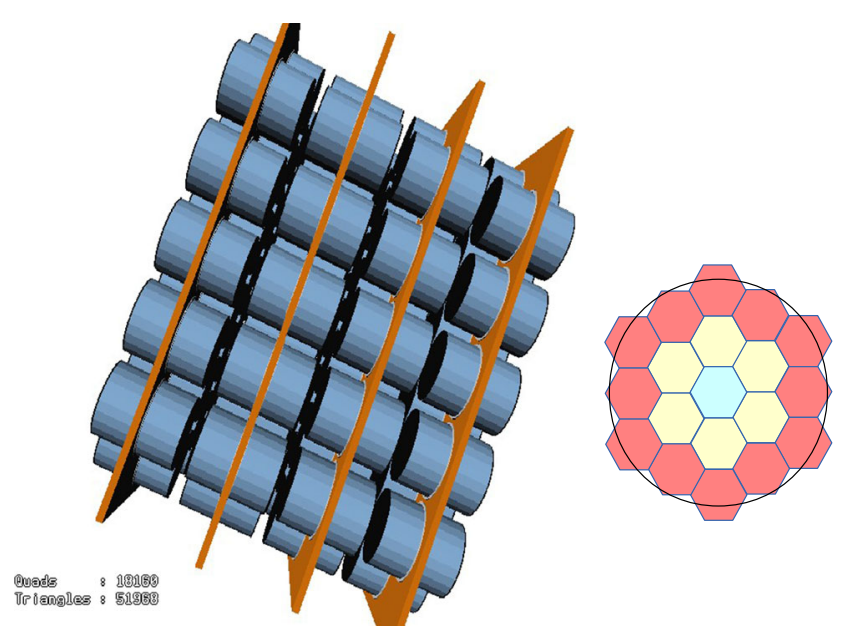

Fig. 4 Array of $108 \mathrm{CaMoO}_{4}$ scintillators (left). Possible configuration of the SDD matrix to be otpically coupled to each circular surface of every detector (right)

surface contaminations of the elements close to the detectors, adequate shielding against environmental sources, and limited exposure to cosmic rays during construction and transportation. The above conceived experimental set-up already addresses many of these requests. A GEANT4-based Monte Carlo simulation of the whole experimental set-up was performed to investigate the effect of the possible background sources on the ROI count rate. In the code, all detector elements can act as a radioactive source with the bulk and surface contaminations independently simulated; for the surfaces, different contamination depths can be chosen. The following possible sources have been considered in the simulation:

- ${ }^{232} \mathrm{Th}$ and ${ }^{238} \mathrm{U}$ bulk contaminations of the $\mathrm{CaMoO}_{4}$ crystals and of the $\mathrm{Cu}$ (holder+tank). Although in the natural radioactive chains there are no intense $\gamma$ lines above $2.615 \mathrm{MeV}$, the presence of the two $\beta$ active isotopes ${ }^{208} \mathrm{Tl}$ and ${ }^{214} \mathrm{Bi}$ - with total decay energies of 4.999 and $3.270 \mathrm{MeV}$, respectively - could result in a substantial contribution to the ROI count rate through coincident $\beta / \gamma$ emissions. Moreover, Bi-Po sub-cascades in both ${ }^{232} \mathrm{Th}$ and ${ }^{238} \mathrm{U}$ chains $-{ }^{212} \mathrm{Bi}\left(\tau_{1 / 2}=60.6 \mathrm{~m}\right) \beta$ decay to ${ }^{212} \mathrm{Po}\left(\tau_{1 / 2}=299 \mathrm{~ns}\right)$, and ${ }^{214} \mathrm{Bi}\left(\tau_{1 / 2}=19.9 \mathrm{~m}\right) \beta$ decay to ${ }^{214} \mathrm{Po}\left(\tau_{1 / 2}=164.3 \mu \mathrm{s}\right)$, respectively - are of even greater concern, since the "quenched" $\alpha$ energy from the Po isotopes sums to the energy of the $\beta$ emitted by the parent $\mathrm{Bi}$ (because of the very short Po half-life), thus contributing with a continuum spectrum to the ROI. The assumed bulk contamination limits for copper are available in [49]. For the $\mathrm{CaMoO}_{4}$ crystals bulk, we have assumed the contamination levels reported in [42]. This is a very conservative assumption since there are promising purification methods of the raw materials needed for the crystal growing which allow to reduce the intrinsic radioactivity of the scintillators, as already demonstrated in [41].

- ${ }^{232} \mathrm{Th}$ and ${ }^{238} \mathrm{U}$ surface contaminations of the $\mathrm{CaMoO}_{4}$ crystals and of the $\mathrm{Cu}$ (holder + tank). Surface contaminations very often exceed the background coming from the bulk of the materials, even in the most radiopure ones: this is supposedly caused by the mechanical and/or chemical treatments of the materials which may contaminate the surfaces or leave them in a highly reactive chemical state. The impact of surface contamination on the background depends on the contaminant and on the depth of the contaminated layer: a depth of $10 \mu \mathrm{m}$ has been considered for both the $\mathrm{CaMoO}_{4}$ crystals and the copper surfaces. The contamination limits for $\mathrm{Cu}$ are taken from [49], while for $\mathrm{CaMoO}_{4}$ we have assumed the same limits of $\mathrm{TeO}_{2}$ in [49].

- ${ }^{232} \mathrm{Th}$ and ${ }^{238} \mathrm{U}$ bulk contaminations of the external $\mathrm{Pb}$. This part of the experimental set-up is placed quite far

Table 6 Monte Carlo simulated contribution of the various background sources to the energy region centered on ${ }^{100}$ Mo $Q_{\beta \beta}$ for the full detector array (108 $\mathrm{CaMoO}_{4}$ detectors). The limits in the last column are obtained by applying off-line rejection analyses, as explained in the text

\begin{tabular}{|c|c|c|c|}
\hline Background source & $\begin{array}{l}\text { Source } \\
\text { activity }\end{array}$ & $\begin{array}{l}\text { Background in ROI } \\
{[\text { counts } /(\mathrm{keV} \cdot \mathrm{kg} \cdot \mathrm{y})]}\end{array}$ & $\begin{array}{l}\text { Background in ROI } \\
\text { (after off-line analysis) } \\
{[\text { counts } /(\mathrm{keV} \cdot \mathrm{kg} \cdot \mathrm{y})]}\end{array}$ \\
\hline${ }^{232} \mathrm{Th}$ in $\mathrm{CaMoO}_{4}$ bulk & $<40 \mu \mathrm{Bq} / \mathrm{kg}$ & $<3 \times 10^{-1}$ & $<3 \times 10^{-4}$ \\
\hline${ }^{238} \mathrm{U}$ in $\mathrm{CaMoO}_{4}$ bulk & $<130 \mu \mathrm{Bq} / \mathrm{kg}$ & $<1 \times 10^{-2}$ & $<1 \times 10^{-5}$ \\
\hline${ }^{232} \mathrm{Th}$ on $\mathrm{CaMoO}_{4}$ surface $(10 \mu \mathrm{m}$ deep $)$ & $<2 \times 10^{-9} \mathrm{~Bq} / \mathrm{cm}^{2}$ & $<8 \times 10^{-6}$ & $<8 \times 10^{-9}$ \\
\hline${ }^{238} \mathrm{U}$ on $\mathrm{CaMoO}_{4}$ surface $(10 \mu \mathrm{m}$ deep $)$ & $<8 \times 10^{-9} \mathrm{~Bq} / \mathrm{cm}^{2}$ & $<1 \times 10^{-6}$ & $<4 \times 10^{-7}$ \\
\hline${ }^{232} \mathrm{Th}$ in $\mathrm{Cu}$ bulk & $<2 \mu \mathrm{Bq} / \mathrm{kg}$ & $<1 \times 10^{-5}$ & \\
\hline${ }^{238} \mathrm{U}$ in $\mathrm{Cu}$ bulk & $<65 \mu \mathrm{Bq} / \mathrm{kg}$ & $<2 \times 10^{-5}$ & \\
\hline${ }^{232} \mathrm{Th}$ on $\mathrm{Cu}$ surface & $<3 \times 10^{-8} \mathrm{~Bq} / \mathrm{cm}^{2}$ & $<8 \times 10^{-5}$ & \\
\hline${ }^{238} \mathrm{U}$ on $\mathrm{Cu}$ surface & $<3 \times 10^{-8} \mathrm{~Bq} / \mathrm{cm}^{2}$ & $<4 \times 10^{-6}$ & \\
\hline${ }^{232} \mathrm{Th}$ in $\mathrm{Pb}$ bulk & $<12 \mu \mathrm{Bq} / \mathrm{kg}$ & $<9 \times 10^{-6}$ & \\
\hline${ }^{238} \mathrm{U}$ in $\mathrm{Pb}$ bulk & $<74 \mu \mathrm{Bq} / \mathrm{kg}$ & $<6 \times 10^{-6}$ & \\
\hline
\end{tabular}


from the detectors, but it may contribute to the ROI count rate through energetic $\gamma \mathrm{s}$ from ${ }^{214} \mathrm{Bi}$ and ${ }^{208} \mathrm{Tl}$ (with very low probabilities) or coincident $\gamma \mathrm{s}$ coming from both natural chains. The probability of such summing becomes negligible as the distance of the emitting source from the detectors increases.

The results of the simulation are presented in Table 6: they have been obtained with the detectors operated in anticoincidence $^{3}$ and by assuming a $\mathrm{QF}=0.2$ for the $\alpha$ particles in $\mathrm{CaMoO}_{4}$. The time resolution of the detectors has been fixed at $1 \mu \mathrm{s}$, the FWHM resolution at (conservatively) $50 \mathrm{keV}$ and the threshold for anticoincidence rejection at $50 \mathrm{keV}$.

The limits reported for the crystals in the last column of Table 6 are obtained with the aid of the $\alpha / \beta$ pulse shape discrimination in $\mathrm{CaMoO}_{4}$ or by exploiting the technique of delayed coincidences within ${ }^{232} \mathrm{Th}$ and ${ }^{238} \mathrm{U}$ radioactive chains. In particular:

1. ${ }^{208} \mathrm{Tl} \beta$ decay $\left(\tau_{1 / 2}=3.05 \mathrm{~m}\right)$ induced background can be strongly reduced by tagging the $\alpha$ particle emitted by the parent nucleus ${ }^{212} \mathrm{Bi}$ ( $\alpha$ decay branching ratio is $36 \%$ ): the dead time resulting by vetoing of the detector for ten half-lives of ${ }^{208} \mathrm{Tl}$ after every ${ }^{212} \mathrm{Bi} \alpha$ decay is negligible $(<0.5 \%)$.

2. The background induced by ${ }^{214} \mathrm{Bi} \beta$ decay (branching ratio $=99.98 \%$ ) can be strongly reduced by tagging the $\alpha$ particle emitted by its daughter nucleus ${ }^{214} \mathrm{Po}$ : the dead time resulting by the removal from the recorded spectrum, of the data segment acquired during the ten ${ }^{214}$ Po half-lives previous to each $\alpha$ decay is completely neglectable.

3. The $\alpha+\beta$ pile-up events of the ${ }^{214} \mathrm{Bi} \rightarrow{ }^{214}$ Po cascades, given the good time resolution of the detectors, can be resolved in $99 \%$ of the cases.

4. Since the ${ }^{212}$ Po half-life is much shorter than the time resolution of the detectors, we have assumed to be able to reject $99 \%$ of the $\alpha+\beta$ pile-up events of the ${ }^{212} \mathrm{Bi} \rightarrow$ ${ }^{212}$ Po cascades thanks to the $\alpha / \beta$ pulse shape discrimination.

As Table 6 shows, with the highly performing detectors proposed in this paper the overall background expected in a ROI of $50 \mathrm{keV}$ centered around the $Q_{\beta \beta}$ of ${ }^{100} \mathrm{Mo}$ is at the extremely encouraging level of $10^{-4}$ counts/(keV·kg.y).

This implies that in the hypothesis of an array of $54 \mathrm{~kg}$ of natural $\mathrm{CaMoO}_{4}$ detectors, the limiting background is

\footnotetext{
3 This configuration allows to reduce the background counts by selecting only the events that deposit all their energy in a single crystal, as it would happen for a $\beta \beta 0 v$ event. Detailed simulations have shown that the containment efficiency of a $\beta \beta 0 v$ event by a $\mathrm{CaMoO}_{4}$ crystal with dimensions similar to those conceived in this paper is $\sim 83 \%$.
}

the unavoidable $\beta \beta 2 v$ continuous spectrum of ${ }^{48} \mathrm{Ca}$ (about $10^{-2}$ counts $\left./(\mathrm{keV} \cdot \mathrm{kg} \cdot \mathrm{y})\right)$. Even in this case, it is thus possible to reach a sensitivity of $\sim 10^{24}$ years on the half-life of the $\beta \beta 0 v$ of ${ }^{100} \mathrm{Mo}$ in only 1 year of data taking at the Gran Sasso Underground Laboratory - to be compared with the presently available limit on ${ }^{100} \mathrm{Mo}\left(1.1 \times 10^{24}\right.$ years [50]).

In the hypothesis of an array of $54 \mathrm{~kg}$ of ${ }^{40} \mathrm{Ca}^{\text {nat }} \mathrm{MoO}_{4}$ crystals (isotopically depleted in ${ }^{48} \mathrm{Ca}$ ) operated in the same conditions, the estimated sensitivity on the half-life of ${ }^{100} \mathrm{Mo}$ $\beta \beta 0 v$ after 1 year of data taking is $\sim 10^{25}$ years. This shows the tremendous potential of the proposed technique.

\section{Other physics measurements with $\mathrm{CaMoO}_{4}$}

A $\beta \beta 0 v$ experiment capable of reaching a high enough sensitivity to deeply explore the IH region of the neutrino mass spectrum will require a mass of the candidate isotope of the order of (or higher than) 1 ton. With an array of $\mathrm{CaMoO}_{4}$ detectors of this size, interesting topics other than $\beta \beta 0 v$ studies may be at reach.

Dark matter may be investigated through the study of the modulation signal. The achievable energy threshold can be evaluated by considering the global light emission of the $\mathrm{CaMoO}_{4}$ crystals operated at low temperatures coupled to the low electronic noise obtainable with the proposed SDD approach. As reported in the previous paragraphs, we estimate a global ENC around $20 e^{-}$rms in normal operating conditions, which means an estimated charge threshold around $100 \mathrm{e}^{-}$considering a Gaussian distribution of the noise fluctuations. Taking into account the photon collection efficiencies reported in Sect. 5, it is possible to estimate an energy threshold of about $6-7 \mathrm{keV}$. This threshold value together with the large detector mass and the low background requested by the $\beta \beta 0 v$ experiment, make the proposed $\mathrm{CaMoO}_{4}$ detector suitable for a competitive Dark Matter experiment.

Neutrino oscillations and neutrino-nucleus interactions can also be studied through the scattering off Mo nuclei. Molybdenum isotopes, in fact, are a good target for neutrino interactions. The first suggestion of using a charged current reaction on ${ }^{100}$ Mo to detect solar neutrinos dates back to the year 2000 [51] with a proposal of using a ${ }^{100}$ Mo based detector (MOON experiment) both for $\beta \beta 0 v$ and real-time solar neutrino studies. Recently charged current neutrino reactions on different Mo isotopes have been discussed [52] in view of their use for the detection of supernova neutrinos or for the study of neutrino-nucleus interactions, as suggested in [53]. As an example of the potentialities, with the cross sections calculated in [52] for neutrinos of $\sim 1 \mathrm{MeV}$ scattering off ${ }^{100}$ Mo nuclei, an interesting interaction rate of $\sim 0.4 \mathrm{day}^{-1}$ is expected already on the $54 \mathrm{~kg}$ natural $\mathrm{CaMoO}_{4}$ crystals array (discussed in Sect. 6) using the nominal rate for a ${ }^{51} \mathrm{Cr}$ 
$v_{e}$ source of $370 \mathrm{PBq}-$ like the one proposed in [54] - at $1 \mathrm{~m}$ distance. Obviously this rate will scale linearly with the number of ${ }^{100}$ Mo nuclei, so a proportionally stronger signal is expected by increasing the detector mass and/or by isotopically enriching the $\mathrm{CaMoO}_{4}$ crystals.

\section{Conclusions}

In this paper we have discussed a new powerful technology for $\beta \beta 0 v$ searches with the potentialities of achieving zero background in the region of interest. Adequate radiopure scintillating crystals coupled to low noise SDD photodetectors result in a detector module with all the demanding characteristics required by future $\beta \beta 0 v$ projects: high energy resolution, low cost mass scalability, flexibility in the choice of the isotope, and powerful tools to make the background negligible. The proposed concept is therefore ideal for exploring the Inverted Hierarchy region of the neutrino masses, since it allows the measurement of very large detector arrays in quite simple experimental setups. The moderate cooling required and the flexibility in the scintillating crystal choice make this approach extremely interesting for many other applications as well. In particular, the $\mathrm{CaMoO}_{4}$ detector array discussed in this work not only can yield competitive results in the $\beta \beta 0 v$ search of ${ }^{100}$ Mo but can also be used for the study of neutrino oscillations and scattering and of dark matter annual modulation, for a high sensitivity multipurpose experiment.

Open Access This article is distributed under the terms of the Creative Commons Attribution License which permits any use, distribution, and reproduction in any medium, provided the original author(s) and the source are credited.

Funded by $\mathrm{SCOAP}^{3}$ / License Version CC BY 4.0.

\section{References}

1. J. Beringer et al., Particle Data Group. Phys. Rev. D 86, 010001 (2012)

2. S.R. Elliott, P. Vogel, Ann. Rev. Nucl. Part. Sci. 52, 115 (2002)

3. F.T. Avignone III et al., Rev. Mod. Phys. 80, 481 (2008)

4. J. Suhonen, O. Civitarese, Phys. Rept. 300, 123 (1998)

5. F. Simkovic et al., Phys. Rev. C 60, 055502 (1999)

6. J. Kotila, F. Iachello, Phys. Rev. C 85, 034316 (2012)

7. J. Menendez et al., Nucl. Phys. A 818, 139 (2009)
8. A. Faessler et al., JoP G Nucl. Part. Phys. 39, 124006 (2012)

9. D. Fang et al., Phys. Rev. C 83, 034320 (2011)

10. J. Suhonen, O. Civitarese, JoP G Nucl. Part. Phys. 39, 124005 (2012)

11. J. Barea et al., Phys. Rev. C 87, 014315 (2013)

12. P.K. Rath et al., Phys. Rev. C 82, 064310 (2010)

13. T.R. Rodriguez et al., Phys. Rev. Lett. 105, 252503 (2010)

14. M. Auger et al., Phys. Rev. Lett. 109, 032505 (2012)

15. A. Gando et al., Phys. Rev. Lett. 110, 062502 (2013)

16. M. Agostini et al., Phys. Rev. Lett. 111, 122503 (2013)

17. O. Cremonesi, M. Pavan, arXiv:1310.4692 (2013)

18. K.H. Ackermann et al., Eur. Phys. J. C73, 2330 (2013)

19. C.E. Aalseth et al., J. Phys. Conf. Ser. 203, 012057 (2010)

20. E. Andreotti et al., Astropart. Phys. 34, 822 (2011)

21. C. Arnaboldi et al., Nucl. Instrum. Meth. 518, 775 (2004)

22. K. Zuber et al., AIP Conf. Proc. 942, 101 (2007)

23. V. Alvarez et al., JINST 7, T06001 (2012)

24. M. Primakov, S.P. Rosen, Ann. Rev. Nucl. Part. Sci. 31, 145 (1981)

25. http://scintillator.lbl.gov/

26. V.B. Mikhailik, H. Kraus, Phys. Status Solidi B 247, 1583 (2007)

27. C. Fiorini et al., IEEE Trans. Nucl. Sci. 60, 2923 (2013)

28. E. Gatti, P.F. Manfredi, Rivista del Nuovo Cimento 9, 1 (1986)

29. R.T. Goldberg et al., in Low Temperature Electronics and High Temperature Superconductivity, ed. by C.L. Claeys, S.I. Raider, W.D. Brown, R.K. Kirschman, PV 95-9, ISBN 1-56677-103-X (1995) p. 428.

30. E. Gatti, P. Rehak, Nucl. Instrum. Meth. 225, 608 (1984)

31. P. Lechner et al., Nucl. Instrum. Meth. A 458, 281 (2001)

32. R.G. Pires et al., Cryogenics 30, 1064 (1990)

33. A. Rachevski et al., Nucl. Instrum. Method A 718, 353 (2013)

34. W. Chen et al., IEEE Nuclear Science Symposium Conference Record N33-4 (2007).

35. C. Fiorini et al., IEEE Trans. Nucl. Sci. 53, 2392 (2006)

36. V.B. Mikhailik et al., Nucl. Instrum. Method A 583, 350 (2007)

37. L. Bardelli et al., Nucl. Instrum. Method A 569, 743 (2006)

38. http://www.crystals.saint-gobain.com

39. G.F. Knoll, Radiation Detection and Measurement (Wiley, New York, 2000) 235.

40. A. Alessandrello et al., Phys. Lett. B 420, 109 (1998)

41. V. Kobychev, Presentation @ RPSCINT 2013 Workshop. http:// lpd.kinr.kiev.ua/rps13/

42. A.N. Annekov et al., Nucl. Instrum. Method A 584, 334 (2008)

43. V.V. Alenkov et al., Cryst. Res. Technol. 46, 1223 (2011)

44. S. Agostinelli et al., Nucl. Instrum. Method A 506, 250 (2003)

45. V. Alenkov et al., JINST 8, P06002 (2013)

46. P. Dorenbos et al., IEEE Trans. Nucl. Sci. 42, 2190 (1995)

47. M. Moszynski et al., Nucl. Instrum. Method A 553, 578 (2005)

48. L.G. Hyman et al., Rev. Sci. Instrum. 35, 393 (1964)

49. D.R. Artusa et al. (CUORE collaboration), arXiv:1404.4469 submitted to EPJ C.

50. A.S. Barabash et al., Phys. Atom. Nucl. 74, 312 (2011)

51. H. Ejiri et al., Phys. Rev. Lett. 85, 2917 (2000)

52. E. Ydrefors et al., Advances in High Energy Physics, vol. 2012 (2012) Article ID 373946.

53. C. Volpe, Nucl. Phys. B (Proc. Suppl.) 143 (2005) 43.

54. G. Bellini et al., JHEP 08, 38 (2013) 